Laser Chem., Vol. 16, pp. 179-196

Reprints available directly from the publisher

Photocopying permitted by license only
(C) 1996 OPA (Overseas Publishers Association) Amsterdam B.V. Published in The Netherlands by Harwood Academic Publishers GmbH

\title{
QUANTUM BEATS IN TRANSIENT SPECTROSCOPY OF J-AGGREGATES
}

\author{
H. GLAESKE, K. -H. FELLER, E. GAISAUKAS**) \\ and L. KNÖLL*)
}

Fachhochschule Jena,

Fachbereich Medizintechnik/Physikalische Technik, Postfach 100314, D-07703 Jena, Germany; *) Friedrich-Schiller-Universität Jena, Theoretisch-Physikalisches Institut, Germany; **) Permanent Address: Laser Research Center, Vilnius University, Lithuania

(Received 6 October, 1995)

\section{Dedicated to Prof. Dr. Alexander Müller (MPI Göttingen) on the occasion of his 65th birthday}

\begin{abstract}
Quantum beats in transient absorption of PIC-J-aggregates, modelled by a modified 3-level-system are calculated. Experimental conditions for the possibility of their observation are investigated. The influence of the temperature dependent phase relaxation on the temporal evolution of quantum beat signals is considered.
\end{abstract}

KEY WORDS: Femtosecond spectroscopy, Coherent oscillations, Quantum beats, J-aggregates, Three-level approximation, Optical Bloch equations.

\section{INTRODUCTION}

Coherent transients in optically excited molecular systems have been in the focus of interest in theoretical as well as experimental work for several years. While earlier investigations of coherent transients concentrated on smaller molecular systems, as for the dephasing times in larger molecular systems are very short, with recent progress in ultrashort laser pulse techniques it is possible to extend the investigations $f$ coherent transients to large molecular systems, too. Among these investigations there were observations of quantum beats as one form of such phenomena (s., e.g.). ${ }^{1,2,3}$ In principle, there are two classes of systems, which are expected to show oscillating behaviour in transient optical experiments:

1. systems with two electronic levels (ground and excited), one of them or both showing a level split off due to vibrational degrees of freedom (see, e.g. ${ }^{4}$ and references therein). Theoretically they can be modelled by three-level systems of the so-called $\Lambda$-type (lower electronic level split off) or V-type (upper electronic level split off) (see, e.g.).). ${ }^{5,6,7}$ Another possibility to model vibrational degrees of freedom is by describing the interference of nuclear wave packets in the wave 
packet picture $[3,4,8,9]$. This type of system configuration is not dealt with in the present paper.

2. systems, which show two excited electronic states, with almost equal transition frequencies between the ground and the first excited and the first and the second excited states. Of this kind are large molecular systems with extended $\pi$-electron systems, and excitations of the excitonic type, for instance polydiacetylenes (PDA) [10], fullerenes [11, 12], and molecular aggregates, such as $\mathrm{J}$-aggregates $[13,14,15,16]$ and the light harvesting complex of chlorophyll [17]. (It is noteworthy, that in the reaction center of the photosynthesis vibrational sublevels are present, so this is an example, where both the features of the class 1 and class 2 systems are present at once). These are the same systems, for which exciton-exciton-annihilation processes are effective as deexciting processes at high excitation densities and compete with the saturation effect [18, 19]. On the other hand, in the last few years, this kind of materials was found to be suitable organic compounds for nonlinear optical (NLO) applications (s. $[20,21,22,23]$ and references therein), because they have large values of the third-order nonlinear susceptibility $\left(\chi^{(3)}\right)$.

In this paper we will deal with a theoretical model for coherent transients in systems of the second type. In former investigations, coherent transients were considered in the framework of a two-level approximation ([24]) (recently extended to a threelevel description to interpret modifications of the pump-probe signal involving twophoton resonance [25].) A calculation of quantum beats in pump-probe signals was performed explicitly by A. Yasuda and T. Kobayashi in [10] using the parameters of PDA as the model system. In that paper a continuous wave was used for probing, and the initial preparation was described by the simplification, that all electrons initially are in the first excited level and no polarization is assumed initially.

On the other hand coherent oscillations with their characteristic time constants of some hundreds of femtosecond could be a very efficient tool for ultrafast alloptical switching in the femtosecond region. Therefore, in the present paper, we discuss the quantum beats created by a superposition of several electronic excited (e.g. exciton) states in molecular systems. We use a three-level model for description of the molecular system, and we describe the preparation of the system by an initial strong ultrashort pump pulse explicitly. The change of the optical density after driving the prepared system by an ultrashort (fs to ps) driving pulse then is tested with a fs probe pulse. As an example for our model calculations in this paper we investigate pseudoisocyanine (PIC)-J-aggregates, which have been proven to be promising third-order NLO-materials, too [26, 27], but the provided model is general for all systems belonging to the second class of materials (see above).

Characteristic features of $\mathbf{J}$-aggregates are a sharp peak in the absorption spectrum, the so called J-band, which is much narrower than and red-shifted from the monomer band, and a strong resonance fluorescence.

The numerical investigations are carried out for PIC-Cl J-aggregates, the parameters of which were taken from the literature [28, 29, 30, 31].

Measurements on PIC-J-aggregates have shown, that the phase relaxation times are strongly temperature dependent. They increase from about $70 \mathrm{fs}$ at room 
temperature up to several ps at $1.5 \mathrm{~K}[29,30]$. Therefore we suppose, that under appropriate conditions, i.e. very low temperatures, quantum beats as a form of coherent transients shold be observable on a time scale comparable to the characteristic time of the energy redistribution by the exciton-exciton annihilation.

The aim of the calculations is to predict the optimum experimental conditions (optimum pulse duration, phase and energy relaxation rate constants etc.) for creating the most pronounced coherent oscillations (e.g. quantum beats).

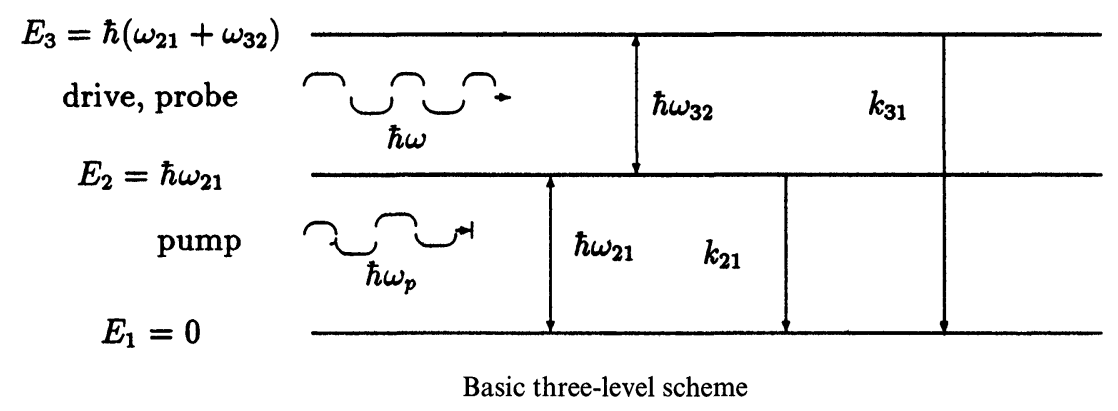

\section{MODEL}

The J-aggregate is modelled by a Basic three-level system with the transition frequencies $\omega_{21}$ and $\omega_{32}$, whereby $\omega_{32} \lesssim \omega_{21}$ (scheme), which is coupled to a heat bath. The interaction with the heat bath is described in relaxation approximation.

The system is prepared by a strong ultrashort laser pulse resonantly coupled to the $|1\rangle \rightarrow|2\rangle$ transition in some initial quantum state and then it is driven by a strong laser pulse that is assumed to be tuned near resonance. The evolution of the system during and after driving is then tested by a weak ultrashort probe pulse of the same frequency as the driving pulse (sequential pump-probe spectroscopy).

There are considered the following processes:

- stimulated emission and absorption induced by the driving field

- longitudinal relaxation from the levels $|3\rangle$ and $|2\rangle$ to the ground state $|1\rangle$ (relaxation constants $\mathrm{k}_{31}$ and $\mathrm{k}_{21}$ ).

- phase relaxation (relaxation constants $\Gamma_{12}, \Gamma_{13}, \Gamma_{23}$ ).

The Hamiltonian of the system is assumed as follows (semiclassical approach, rotating wave approximation):

$$
\begin{aligned}
\hat{\mathrm{H}} & =\hat{\mathrm{H}}_{\text {sys }}+\hat{\mathrm{H}}_{\text {drive }}^{\text {(RWA }} \\
\hat{\mathrm{H}}_{\text {sys }} & =\hbar \omega_{21} \widehat{\mathrm{A}}_{22}+\hbar\left(\omega_{21}+\omega_{32}\right) \widehat{\mathrm{A}}_{33} \\
\hat{\mathrm{H}}_{\text {drve }}^{(\mathrm{RWA})} & =-\left(\mathrm{d}_{21} \overrightarrow{\mathrm{e}} *(\mathrm{t}) \widehat{\mathrm{A}}_{21}+\mathrm{d}_{32} \overrightarrow{\mathrm{e}} *(\mathrm{t}) \widehat{\mathrm{A}}_{32}+\{\mathrm{H} . \mathrm{A} .\}\right),
\end{aligned}
$$

where

$$
\widehat{\mathrm{A}}_{\mathrm{ij}}=|\mathrm{i}\rangle\langle\mathrm{j}|
$$


are atomic flip operators,

$$
\overrightarrow{\mathrm{E}}_{\text {drive }}(\mathrm{t})=\overrightarrow{\mathrm{e}}(\mathrm{t})+\{\text { c. c. }\}=\overrightarrow{\mathrm{e}}\left(\epsilon(\mathrm{t}) \mathrm{e}^{\mathrm{i} \omega \mathrm{t}}+\epsilon^{*}(\mathrm{t}) \mathrm{e}^{-\mathrm{i} \omega \mathrm{t}}\right)
$$

is the driving field, $\in(t)$ describes the pulse shape, and $d_{i j}$ are the transition dipole moments.

The equation of motion of the density operator of the atomic system in relaxation approximation then is

$$
\dot{\hat{\rho}}=(\dot{\hat{\rho}})_{\mathrm{dyn}}+(\dot{\hat{\rho}})_{\mathrm{rel}}
$$

the dynamic part being

$$
(\dot{\hat{\rho}})_{\mathrm{dyn}}=\frac{\mathrm{i}}{\hbar}[\hat{\rho}, \hat{\mathrm{H}}]
$$

After introducing the density matrix elements according to

$$
\hat{\rho}=\sum_{\mathrm{i}, \mathrm{j}} \rho_{\mathrm{ij}}|\mathrm{i}\rangle\langle\mathrm{j}|
$$

we obtain in relaxation approximation with the above mentioned relaxation constants being nonzero the following system of differential equations:

$$
\begin{gathered}
\dot{\rho}_{11}=\mathrm{k}_{21} \rho_{22}+\mathrm{k}_{31} \rho_{33}-\mathrm{i} \Omega_{12}^{*}(\mathrm{t}) \tilde{\rho}_{12}+\mathrm{i} \Omega_{12}(\mathrm{t}) \tilde{\rho}_{21} \\
\dot{\rho}_{22}=-\mathrm{k}_{21} \rho_{22}+\mathrm{i} \Omega_{12}^{*}(\mathrm{t}) \tilde{\rho}_{12}-\mathrm{i} \Omega_{12}(\mathrm{t}) \tilde{\rho}_{21} \\
-\mathrm{i} \Omega_{23}^{*}(\mathrm{t}) \tilde{\rho}_{23}+\mathrm{i} \Omega_{23}(\mathrm{t}) \tilde{\rho}_{32} \\
\dot{\rho}_{33}=-\mathrm{k}_{31} \rho_{33}+\mathrm{i} \Omega_{23}^{*}(\mathrm{t}) \tilde{\rho}_{23}-\mathrm{i} \Omega_{23}(\mathrm{t}) \tilde{\rho}_{32} \\
\dot{\tilde{\rho}}_{12}=\left(-\mathrm{i} \delta_{1}-\Gamma_{12}\right) \tilde{\rho}_{12}-\mathrm{i} \Omega_{23}^{*}(\mathrm{t}) \tilde{\rho}_{13}+\mathrm{i} \Omega_{12}(\mathrm{t}) \rho_{22}-\mathrm{i} \Omega_{12}(\mathrm{t}) \rho_{11} \\
\dot{\tilde{\rho}}_{13}=\left(-\mathrm{i}\left(\delta_{1}+\delta_{2}\right)-\Gamma_{13}\right) \tilde{\rho}_{13}+\mathrm{i} \Omega_{12}(\mathrm{t}) \tilde{\rho}_{23}-\mathrm{i} \Omega_{23}(\mathrm{t}) \tilde{\rho}_{12} \\
\dot{\tilde{\rho}}_{23}=\left(-\mathrm{i} \delta_{2}-\Gamma_{23}\right) \tilde{\rho}_{23}+\mathrm{i} \Omega_{12}^{*}(\mathrm{t}) \tilde{\rho}_{13}-\mathrm{i} \Omega_{23}(\mathrm{t}) \rho_{22}+\mathrm{i} \Omega_{23}(\mathrm{t}) \rho_{33} \\
\tilde{\rho}_{21}=\tilde{\rho}_{12}^{*}, \quad \tilde{\rho}_{31}=\tilde{\rho}_{13}^{*}, \quad \tilde{\rho}_{32}=\tilde{\rho}_{23}^{*} .
\end{gathered}
$$

The slowly varying non-diagonal matrix elements are introduced as follows:

$$
\begin{gathered}
\tilde{\rho}_{12}=\mathrm{e}^{-\mathrm{i} \omega \mathrm{t}} \rho_{12} \\
\tilde{\rho}_{23}=\mathrm{e}^{-\mathrm{i} \omega \mathrm{t}} \rho_{23} \\
\tilde{\rho}_{13}=\mathrm{e}^{-2 \mathrm{i} \omega t} \rho_{13}
\end{gathered}
$$

The detunings of the laser frequency from the transition frequencies are $\delta_{1}=\omega-\omega_{21}$ and $\delta_{2}=\omega-\omega_{32}$. The "time dependent Rabi frequencies" are defined as

$$
\Omega_{\mathrm{ij}}(\mathrm{t})=\frac{\overrightarrow{\mathrm{d}}_{\mathrm{ij}} \overrightarrow{\mathrm{e}} \in(\mathrm{t})}{\hbar}
$$

For the observation of optical quantum beats in J-aggregates a setup of three pulses is proposed. The first pulse (pump pulse) is to prepare the initial state of the system. 
The second pulse is a strong external driving pulse that is applied just after pumping and the duration of which, $t_{\text {drive }}$, can be varied from about 100 fs to some ps. The dynamical evolution of the system under the influence of this pulse is described by equations $(2.9-2.15)$.

The third pulse (probe pulse) is to probe the state of the driven system by measuring its transmission in dependence on the delay time between the third and the second pulse. Therefore it has to be as short as possible and should be much weaker than the driving pulse for the neglect of its influence on the system evolution.

The quantity to be measured is the change of the energy of the transmitted probe pulse,

$$
\mathrm{W}=-\int \overline{\overrightarrow{\mathrm{E}}_{\text {probe }}\left(\mathrm{t}, \mathrm{t}_{\mathrm{D}}\right) \dot{\overrightarrow{\mathrm{p}}}(\mathrm{t})} \mathrm{dt},
$$

where $\vec{E}_{\text {probe }}$ is the electric field of the probe pulse. It is defined in analogy to the driving field (2.5), and the phase is assumed locked to the driving field. $\overrightarrow{\mathrm{E}}_{\text {probe }}$ depends on the delay time $t_{D}$ (the time difference between the peak of the probe pulse and the peak of the driving pulse) as a parameter. $\vec{p}(t)$ is the dipole moment induced by the driving field, and the bar means time averaging over one period of the field.

According to the definition of the dipole moment of an atomic system

$$
\overrightarrow{\mathrm{p}}(\mathrm{t})=\operatorname{Tr}(\hat{\rho} \hat{\mathrm{d}})
$$

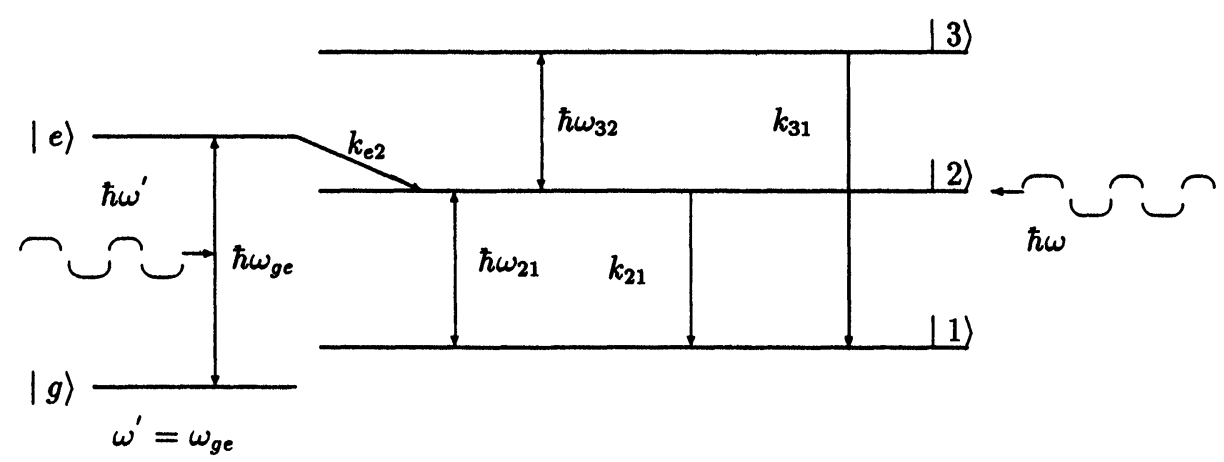

Modified three-level scheme

we obtain

$$
\dot{\overrightarrow{\mathrm{p}}}(\mathrm{t})=\dot{\rho}_{12} \overrightarrow{\mathrm{d}}_{12}^{*}+\dot{\rho}_{21} \overrightarrow{\mathrm{d}}_{12}+\dot{\rho}_{32} \overrightarrow{\mathrm{d}}_{23}+\dot{\rho}_{23} \overrightarrow{\mathrm{d}}_{23}^{*},
$$

and, after again introducing the slowly varying amplitudes

$$
\begin{aligned}
\overline{-\dot{\mathrm{p}}_{\mathrm{E}}^{\text {probe }}}= & -\hbar\left[\left(\dot{\tilde{\rho}}_{21}-\mathrm{i} \omega \tilde{\rho}_{21}\right) \Omega_{12, \text { probe }}+\left(\dot{\tilde{\rho}}_{12}+\mathrm{i} \omega \tilde{\rho}_{12}\right) \Omega_{12, \text { probe }}^{*}\right. \\
& \left.+\left(\dot{\tilde{\rho}}_{32}-\mathrm{i} \omega \tilde{\rho}_{32}\right) \Omega_{23, \text { probe }}+\left(\dot{\tilde{\rho}}_{23}+\mathrm{i} \omega \tilde{\rho}_{23}\right) \Omega_{23, \text { probe }}^{*}\right]
\end{aligned}
$$

with $\Omega_{\mathrm{ij} \text {, probe }}\left(\mathrm{t}, \mathrm{t}_{\mathrm{p}}\right)$ defined analogously to $\Omega_{\mathrm{ij}}(\mathrm{t})(2.19)$, by exchanging the characteristics of the driving pulse by those of the probe pulse. 
So, by solving the system (2.9-2.15), the integrand (2.23) can be easily calculated, and after integration it is obtained (2.20).

The effect of the pump pulse on the three-level-system can be modelled in several ways.

The simplest one is, to assume, that at time 0 , before the beginning of the driving pulse, the system was prepared in the pure state $|2\rangle$. This means the assumption of a $\delta$-shaped pump pulse, and is equivalent to solving the equations of motion $(2.9-2.15)$ with the initial conditions

$$
\left.\begin{array}{l}
\rho_{22}(0)=1 \\
\rho_{\mathrm{ij}}(0)=0,(\mathrm{ij}) \neq(22) .
\end{array}\right\}
$$

Our model calculations show, that under such an assumption, if only one of the levels is excited initially, the generation of quantum beats is less effective than for more general initial conditions.

More generally, the preparation of the state of the system can be modelled by describing the action of a pump pulse of finite time duration on the three-level system initially being in the ground state $|1\rangle$. If the pump pulse is assumed to be ultrashort (up to several hundreds of femtoseconds of duration) the relaxation processes during its action can be neglected, and it is possible to describe the system by the probability amplitudes $a_{i}$ of the states $|i\rangle$. The density matrix elements are defined in terms of the $\mathrm{a}_{\mathrm{i}}$ according to $\rho_{\mathrm{ij}}=\mathrm{a}_{\mathrm{i}} \mathrm{a}_{\mathrm{j}}^{*}$. If there is assumed exact resonance between the pump pulse and the "lower" transition, the frequency of the pump pulse being $\omega_{\mathrm{p}}=\omega_{21}$, and there are assumed slowly varying amplitudes according to

$$
\begin{aligned}
& \mathrm{a}_{1}=\tilde{\mathrm{a}}_{1}, \\
& \mathrm{a}_{2}=\tilde{\mathrm{a}}_{2} \mathrm{e}^{-\mathrm{i} \omega_{\mathrm{p}} \mathrm{t}}, \\
& \mathrm{a}_{3}=\tilde{\mathrm{a}}_{3} \mathrm{e}^{-2 \mathrm{i} \omega_{\mathrm{p}} \mathrm{t}},
\end{aligned}
$$

the system of equations for the amplitudes is

$$
\begin{aligned}
& \dot{\tilde{\mathrm{a}}}_{1}=\mathrm{i} \Omega_{12, \text { pump }} \tilde{\mathrm{a}}_{2}, \\
& \dot{\tilde{\mathrm{a}}}_{2}=\mathrm{i} \Omega_{12, \text { pump }}^{*} \tilde{\mathrm{a}}_{1}+\mathrm{i} \Omega_{23, \text { pump }} \tilde{\mathrm{a}}_{3}, \\
& \dot{\tilde{\mathrm{a}}}_{3}=\mathrm{i} \Delta_{2} \tilde{\mathrm{a}}_{3}+\mathrm{i} \Omega_{23, \text { pump }}^{*} \tilde{\mathrm{a}}_{2},
\end{aligned}
$$

with $\Delta_{2}=\omega_{p}-\omega_{32}$ and $\Omega_{\mathrm{ij}, \text { pump }}(\mathrm{t})$ being defined analogously to $\Omega_{\mathrm{ij}}(\mathrm{t})$ (2.19), by exchanging the characteristics of the driving pulse by those of the pump pulse.

It has to be solved with the initial conditions

$$
\left.\mathrm{a}_{1}\left(\mathrm{t}_{0}\right)=1, \quad \mathrm{a}_{2}\left(\mathrm{t}_{0}\right)=0=\mathrm{a}_{3}\left(\mathrm{t}_{0}\right)\right\}
$$

for $t_{0}<0$; $t_{0}$ being the time of the beginning of the pump pulse. The density matrix elements calculated from the amplitudes for $t=0$ after the pump pulse has passed are used then as generalized initial conditions instead of (2.24).

Finally, with respect to possible generalizations of our model to other molecular systems as an alternative we consider the following description of the pump process 
(see modified three-level scheme). The three-level system is assumed to be coupled to another two-level system by a (fast) transfer process from the excited level of the two-level system $|e\rangle$, to the second level of the three-level system, $|2\rangle$. Therefore $|e\rangle$ is considered to have a slightly higher energy than $|2\rangle$. The pump pulse of frequency $\omega^{\prime}$ is in resonance with the $\omega^{\prime}=\omega_{\text {eg }}$ transition $|\mathrm{g}\rangle \rightarrow|\mathrm{e}\rangle$ from the two-level ground to the excited state. The modifications necessary compared with the model described above are as follows. Equation (2.10) has to be replaced by

$$
\dot{\rho}_{22}=\mathrm{k}_{\mathrm{e} 2} \rho_{\mathrm{ee}}-\mathrm{k}_{21} \rho_{22}+\mathrm{i} \Omega_{12}^{*}(\mathrm{t}) \tilde{\rho}_{12}-\mathrm{i} \Omega_{12}(\mathrm{t}) \tilde{\rho}_{21}-\mathrm{i} \Omega_{23}^{*}(\mathrm{t}) \tilde{\rho}_{23}+\mathrm{i} \Omega_{23}(\mathrm{t}) \tilde{\rho}_{32},
$$

where $\mathrm{k}_{\mathrm{e} 2}$ is the transfer constant from $|\mathrm{e}\rangle$ to $|2\rangle$. The time dependent population of the level $|\mathrm{e}\rangle, \rho_{\mathrm{ee}}$, is calculated from the system of density matrix equations for the two-level system,

$$
\begin{aligned}
\dot{\rho}_{\mathrm{ee}} & =-\mathrm{i} \Omega_{\mathrm{ge}}(\mathrm{t}) \mathrm{v}-\mathrm{k}_{\mathrm{e} 2} \rho_{\mathrm{ee}} \\
\dot{\mathrm{v}} & =2 \mathrm{i} \Omega_{\mathrm{ge}}(\mathrm{t})\left(\rho_{\mathrm{gg}}-\rho_{\mathrm{ee}}\right)-\mathrm{k}_{\mathrm{e} 2 \mathrm{v}} \\
\dot{\rho}_{\mathrm{gg}} & =\mathrm{i} \Omega_{\mathrm{ge}}(\mathrm{t}) \mathrm{v}
\end{aligned}
$$

with $\mathrm{v}=\tilde{\rho}_{\mathrm{eg}}-\tilde{\rho}_{\mathrm{ge}}$.

Under the assumptions of fast transfer, $\mathrm{k}_{\mathrm{e} 2} \mathrm{v} \gg \dot{\mathrm{v}}$, and weak pumping, $\rho_{\mathrm{gg}}-\rho_{\mathrm{ee}} \approx 1$, equation (2.33) reduces to

$$
\dot{\rho}_{\mathrm{ee}}=2 \frac{\Omega_{\mathrm{ge}}^{2}(\mathrm{t})}{\mathrm{k}_{\mathrm{e} 2}}-\mathrm{k}_{\mathrm{e} 2} \rho_{\mathrm{ee}} .
$$

With the initial condition $\rho_{\mathrm{ee}}(-\infty)=0$ and assuming a double exponential shape of the pump pulse,

$$
\Omega_{\mathrm{ge}}^{2}(\mathrm{t})=\kappa \exp \left(-\frac{|\mathrm{t}|}{\tau}\right),
$$

the square root of the constant $\kappa$ being the maximum Rabi frequency of the pump process, (2.36) can be solved analytically to obtain

$$
\rho_{\mathrm{ee}}(\mathrm{t})= \begin{cases}2 \frac{\kappa}{\mathrm{k}_{\mathrm{e} 2}\left(\mathrm{k}_{\mathrm{e} 2}+\frac{1}{\tau}\right)} \exp (\mathrm{t} / \tau), & \mathrm{t}<0 \\ \frac{\kappa}{\mathrm{k}_{\mathrm{e} 2}\left(\mathrm{k}_{\mathrm{e} 2}-\frac{1}{\tau}\right)} \exp (-\mathrm{t} / \tau)-\frac{4 \kappa}{\mathrm{k}_{\mathrm{e} 2} \tau\left(\mathrm{k}_{\mathrm{e} 2}^{2}-\frac{1}{\tau}\right)^{2}} \exp \left(-\mathrm{k}_{\mathrm{e} 2} \mathrm{t}\right) & \mathrm{t}>0\end{cases}
$$

With this solution as an input the system $(2.9,2.11-2.15,2.32)$ has to be solved, in this case with zero initial conditions.

Such a modification of our model was considered with respect to possible generalizations to other molecular systems than J-aggregates.

\section{RESULTS AND DISCUSSION}

For our numerical calculations we have considered the preparation by the pump pulse explicitly, i.e. we have used the second of the three possibilities to introduce 
the initial conditions we have outlined in section 2. For the calculations we used the following values of the relevant parameters: $\omega_{12}=3.289 \times 10^{15} \mathrm{~s}^{-1} \hat{=} 573 \mathrm{~nm}$, $\omega_{23}=3.2782 \times 10^{15} \mathrm{~s}^{-1} \hat{=} 575 \mathrm{~nm}, \mathrm{k}_{21}^{-1}=70 \mathrm{ps}, \mathrm{k}_{31}^{-1}=5 \mathrm{ps}, \Gamma_{12}^{-1}=\Gamma_{13}^{-1}=\Gamma_{23}^{-1}=9 \mathrm{ps}$. It is well known from experimental results $[29,30]$, that these values are characteristic for PIC-J-aggregates at very low temperatures $(\sim 1.5 \mathrm{~K})$. The other parameters are given in the inserts of the figures in units of $\mathrm{ps}$ and $\mathrm{ps}^{-1}$, i.e. THz. $\Omega_{\mathrm{ij}}$ means the maximum value of the driving pulse Rabi frequency, $\Omega_{\mathrm{ij}}(\mathrm{t})$. The Rabi frequency of the probe pulse is assumed to be 0.2 times the Rabi frequency of the driving pulse.

The pump pulse is assumed to have a Gaussian shape, and the peak is situated at $\mathrm{t}=-\mathrm{t}_{\text {pump }}$. By solving the system $(2.28-2.30)$ under (2.31) it can be seen, that a wide variety of initial conditions for $(2.9-2.15)$ can be generated by the proper choice of the pump pulse. The most characteristic features are displayed, if we use pulses of an area of integer and half integer times $\pi$. Figures 1 and 2 show, that for fairly long (the duration of the pump pulse being $\mathrm{t}_{\text {pump }}=4 \mathrm{ps}$ ) $\pi$ - and $2 \pi$-pulses we obtain a situation similar to a two-level system, the upper level of $|3\rangle$ not being involved at all. For the $\pi$-pulse, we get a full occupation inversion, i.e. the initial condition (2.24), and after the $2 \pi$-pulse the initial state (2.31) is obtained again. Figures 3,4 and 5 show the influence of $\pi / 2-, \pi$ - and $2 \pi$-pulses of ultrashort duration and high intensity $\left(t_{\text {pump }}=130 \mathrm{fs}\right.$ FWHM). In this case all three levels are involved in the pump process, and it is possible to reach a situation after pumping, where the occupation numbers of all three levels are of the same magnitude, and so are the polarization components $\rho_{12}, \rho_{23}$, and $\rho_{13}$ (in the case of the $2 \pi$-pulse, and even more for a pulse area of $2.11 \pi$ (fig.6)).

In this case the generation of quantum beats in the differential absorption of a probe pulse after driving is most efficient.

Therefore we used the initial conditions according to fig. 6 for the most of our numerical examples in this paper. In an experiment the pump pulse of this type can be obtained by using a Ti-Sapphire laser after 5 -fold amplification. Our calculations of the quantum beats are performed for a series of different central frequencies $\omega$ of the Gaussian driving pulse, which is centered at $\mathrm{t}=\mathrm{t}_{\text {drive }}$. This leads to different values of the detunings $\delta_{1}$ and $\delta_{2}$, starting from $\left(\delta_{1}<\delta_{2}<0\right)$ through $\left(\delta_{1}<0 \leqslant \delta_{2}\right)$ up to $\left(0 \leqslant \delta_{1}<\delta_{2}\right)$. As can be seen from the curves of figures 7 to 13 , under appropriate experimental conditions the optical density change, furthermore called differential absorption, in dependence on the delay time $t_{D}$ of the ultrashort $(100-200 \mathrm{fs})$ probe pulse shows damped oscillations with the detuning frequency of the "more resonant" transition, i.e. the frequency $\delta_{\mathrm{i}}$ with $\left|\delta_{\mathrm{i}}\right|<\left|\delta_{\mathrm{j}}\right|,(\mathrm{i}, \mathrm{j}=1 ; 2)$. The amplitude of these oscillations is modulated with the other detuning frequency, $\delta_{\mathrm{j}}$. This is explained, if we remember, that for delay times $t_{D} \gg t_{\text {drive, }},\left(t_{\text {drive }}\right.$ being the duration of the driving pulse), when $\Omega_{\mathrm{ij}}=0$ holds, the equations of motion decouple, and we have the situation of free time evolution of the occupation number and polarization matrix elements. In this case we obtain

$$
\begin{aligned}
& \tilde{\rho}_{12}=\tilde{\rho}_{12}(\mathrm{~T}) \mathrm{e}^{\left(-\mathrm{i} \delta_{1}-\Gamma_{12}\right)(\mathrm{t}-\mathrm{T})} \\
& \tilde{\rho}_{13}=\tilde{\rho}_{13}(\mathrm{~T}) \mathrm{e}^{\left(-1\left(\delta_{1}+\delta_{2}\right)-\Gamma_{13}\right)(\mathrm{t}-\mathrm{T})} \\
& \tilde{\rho}_{23}=\tilde{\rho}_{23}(\mathrm{~T}) \mathrm{e}^{\left(-\mathrm{i} \delta_{2}-\Gamma_{23}\right)(\mathrm{t}-\mathrm{T})}, \mathrm{t}>\mathrm{T},
\end{aligned}
$$




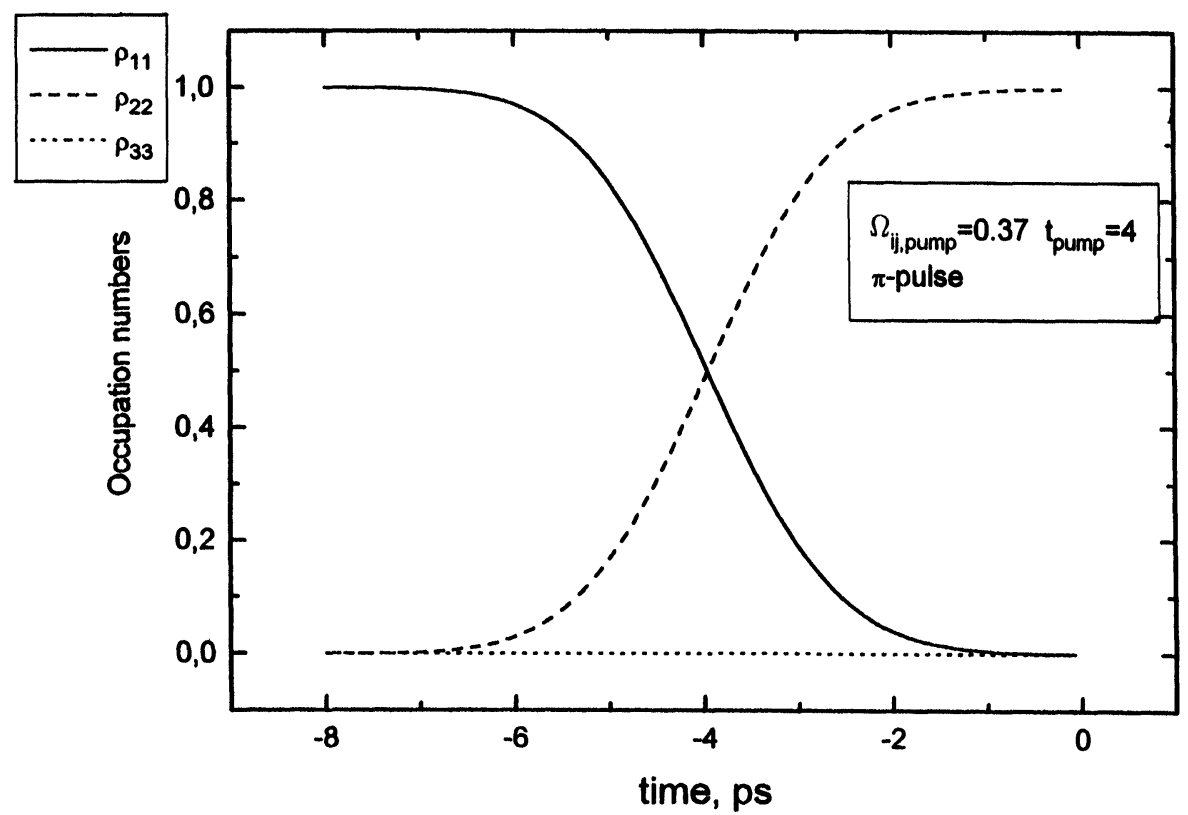

Figure 1 Evolution of the occupation numbers under the influence of a $4 \mathrm{ps} \pi$-pulse, other parameters see insert.

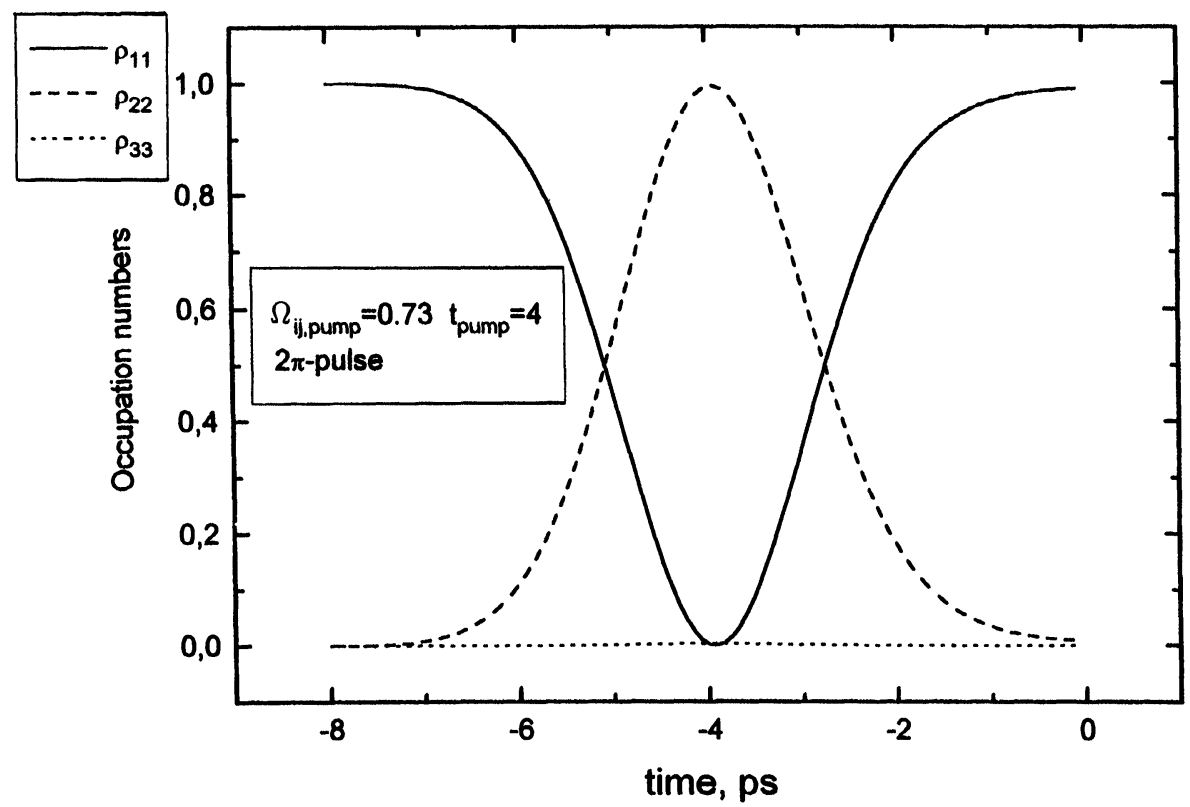

Figure 2 Evolution of the occupation numbers under the influence of a $4 \mathrm{ps} 2 \pi$-pulse, other parameters see insert. 


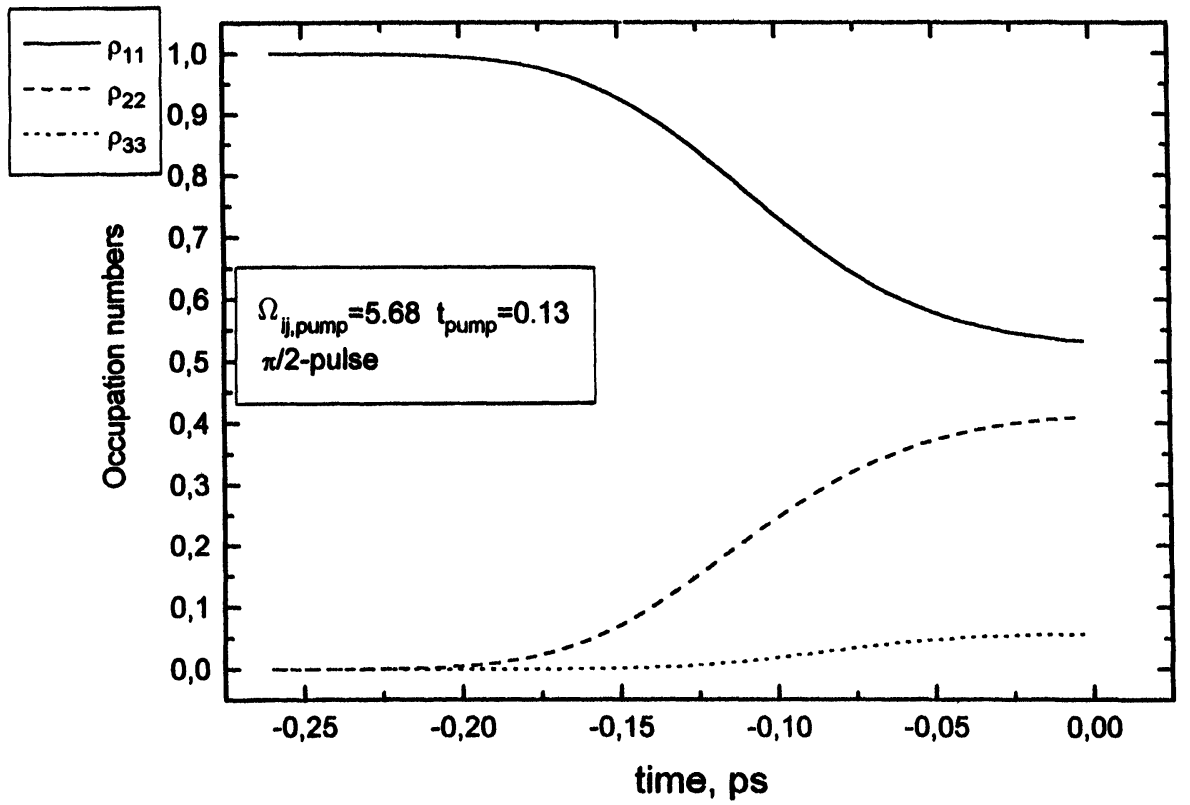

Figure 3 Evolution of the occupation numbers under the influence of a $130 \mathrm{fs} \pi / 2$-pulse, other parameters see insert.

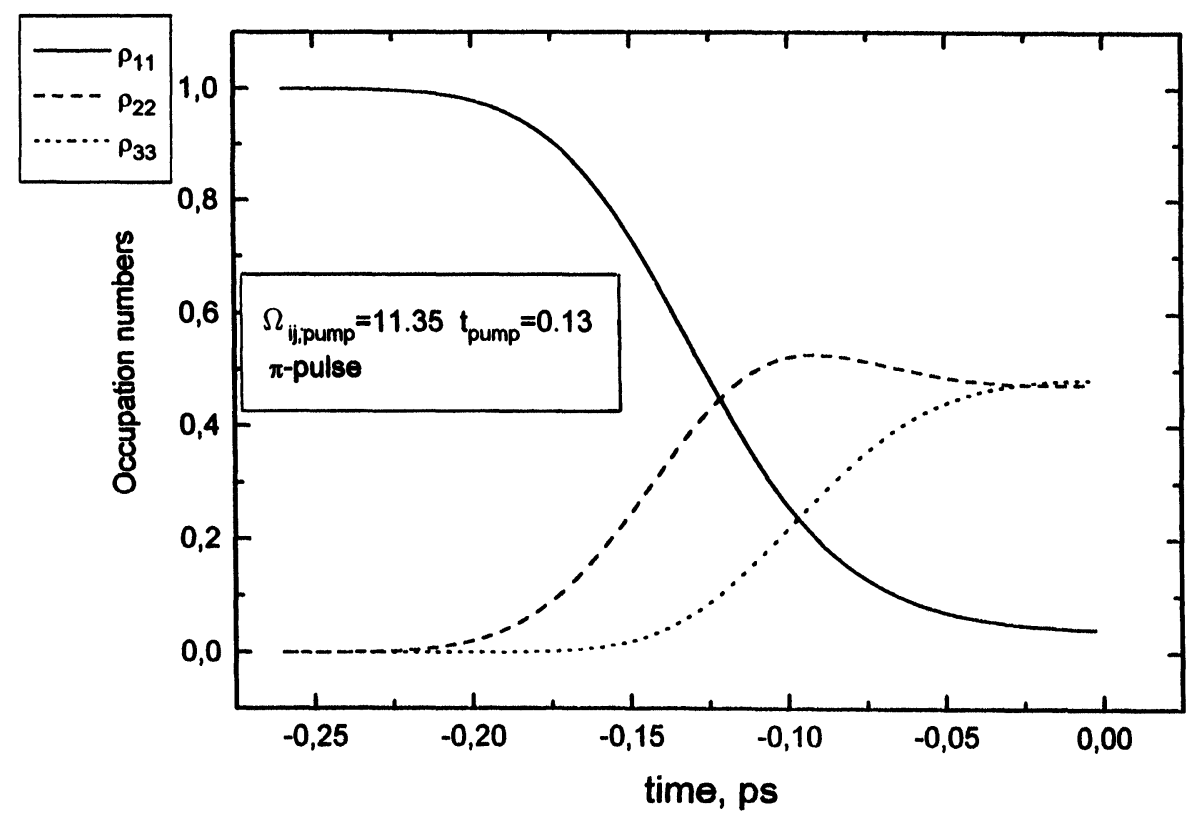

Figure 4 Evolution of the occupation numbers under the influence of a 130fs $\pi$-pulse, other parameters see insert. 


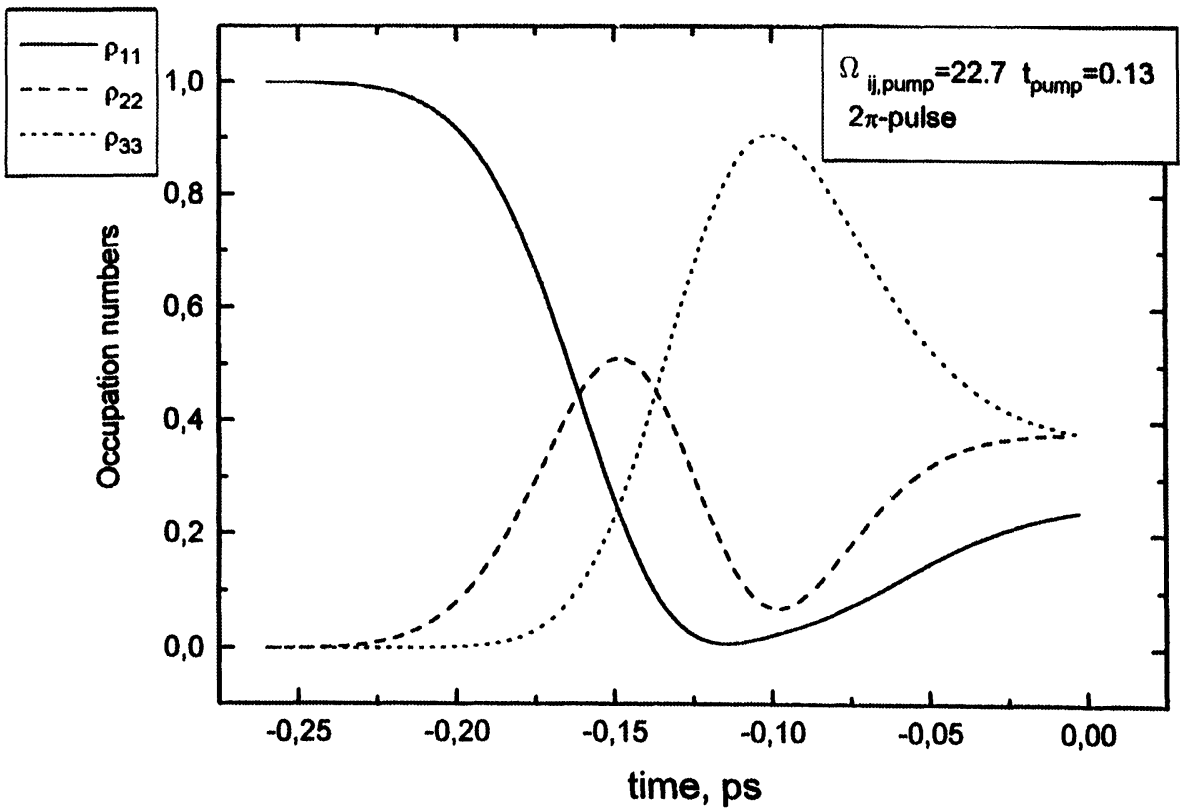

Figure 5 Evolution of the occupation numbers under the influence of a 130fs $2 \pi$-pulse, other parameters see insert.

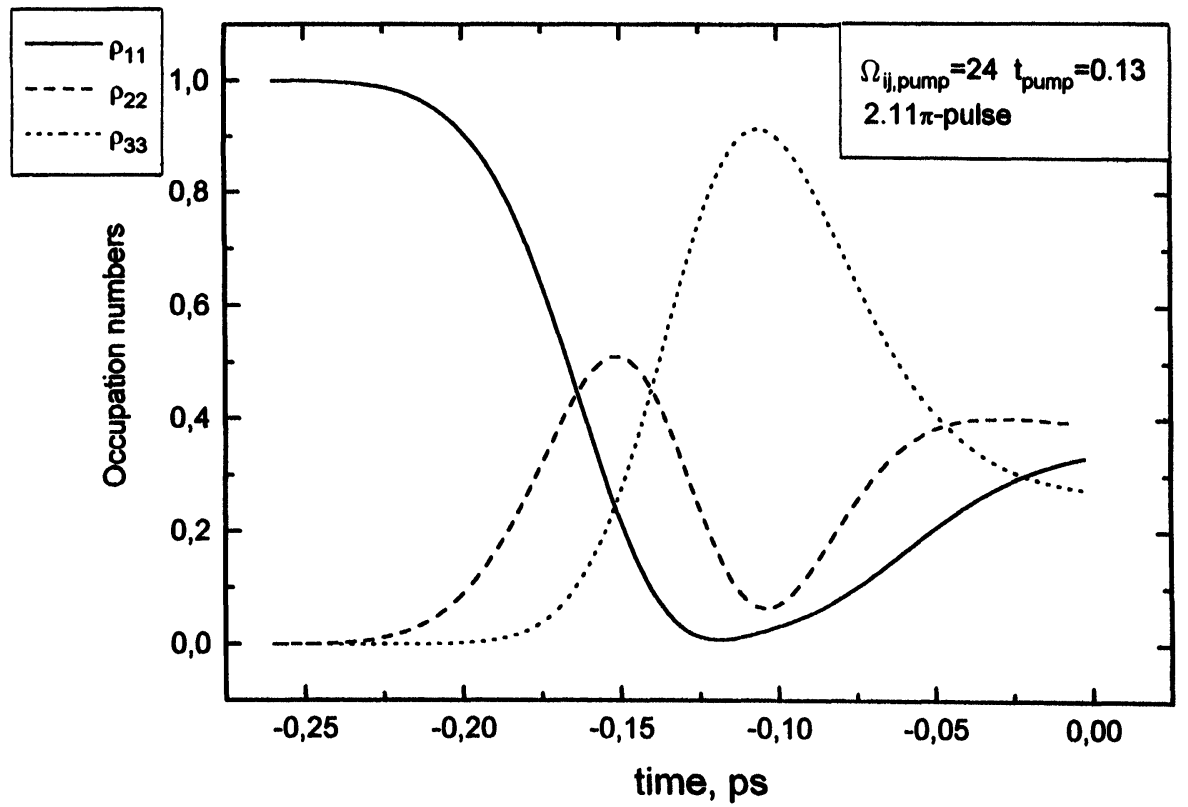

Figure 6 Evolution of the occupation numbers under the influence of a $130 \mathrm{fs} 2.11 \pi$-pulse, other parameters see insert. 
with an appropriate chosen "initial time of the free evolution" $T$, being the time at the end of the driving pulse.

We then get

$$
\begin{aligned}
\overline{-\dot{\overrightarrow{\mathrm{p}}} \overrightarrow{\mathrm{E}}_{\text {probe }}}= & -2 \hbar \operatorname{Re}\left[\tilde{\rho}_{12}^{*}(\mathrm{~T})\left[-\mathrm{i} \omega_{21}-\Gamma_{12}\right]\right) \mathrm{e}^{\left(-\mathrm{i} \delta_{1}-\Gamma_{12}\right)(\mathrm{t}-\mathrm{T})} \Omega_{12, \text { probe }} \\
& \left.+\tilde{\rho}_{23}^{*}(\mathrm{~T})\left[-\mathrm{i} \omega_{32}-\Gamma_{23}\right] \mathrm{e}^{\left(-\mathrm{i} \delta_{2}-\Gamma_{23}\right)(\mathrm{t}-\mathrm{T})} \Omega_{23, \text { probe }}\right]
\end{aligned}
$$

i.e. the differential absorption signal is expected to be a superposition of damped oscillations with the frequencies $\delta_{1}$ and $\delta_{2}$ and amplitudes depending on the "initial values" $\tilde{\rho}_{12}(\mathrm{~T})$ and $\tilde{\rho}_{23}(\mathrm{~T})$.

Now we will discuss some special cases for the parameters. The signal is shown in arbitrary units, set to zero for minimum delay time, and the maximum absolute value is normalized to unity. Fig. 7 shows the differential absorption for a typical parameter set in the range $\left(\delta_{1}<0, \delta_{2}>0\right)$. The calculations are performed for $\Omega_{12}=\Omega_{23}$. We have shown, that there is no significant influence on the absorption curves, if we use different values for the maximum Rabi frequencies for the two transitions, in the range of driving conditions we have used.

In fig. 8 the frequency values have been changed:

$$
\delta_{1}=11 \mathrm{ps}^{-1} \approx 10 \mathrm{ps}^{-1},
$$

but

$$
\delta_{2}=0.4 \mathrm{ps}^{-1} \ll 1.4 \mathrm{ps}^{-1} .
$$

The modification of the main frequency is evident. The amplitude of the main oscillation decreases in the phase relaxation time of $9 \mathrm{ps}$.

An alteration of the maximum Rabi frequency $\Omega_{\mathrm{ij}}$ does not change the picture significantly.

Fig. 9 shows an example, in which the modules of $\delta_{1}$ and $\delta_{2}$ are interchanged with respect to fig. 8 . This results in a change of the sign of the differential absorption at zero delay.

Fig. 10 is an example for a longer driving time, $\mathrm{t}_{\text {drive }}=4 \mathrm{ps}$. The amplitude modulations are much less pronounced. Our calculations show, that this is due to a breakdown of $\tilde{\rho}_{12}$ as a result of stimulated emission processes during the longer pulse, and therefore after the driving it holds $\left|\tilde{\rho}_{23}\right| \gg\left|\tilde{\rho}_{12}\right|$, and one oscillation component in (3.4) contributes much more to the signal than the other one.

This is even more pronounced in the case of the initial conditions (2.24). As can be seen by comparison of fig. 11 and fig. 12, for ps driving pulses then the amplitude modulations are no longer observable at all.

In fig. 13 it is shown the situation, when both the detunings are almost equal. As for the amplitudes are almost equal then as well, we see oscillations with the detuning frequency $\left|\delta_{1}\right| \approx \delta_{2}$, the amplitude of which breaks down and builds up again with the difference $\| \delta_{1}\left|-\delta_{2}\right|$.

Our main results concerning the experimental conditions necessary for the observation of quantum beats are as follows:

a) Quantum beats in sequential pump-probe spectroscopy should be observable in systems, such as J-aggregates, in which may occur absorption and stimulated 


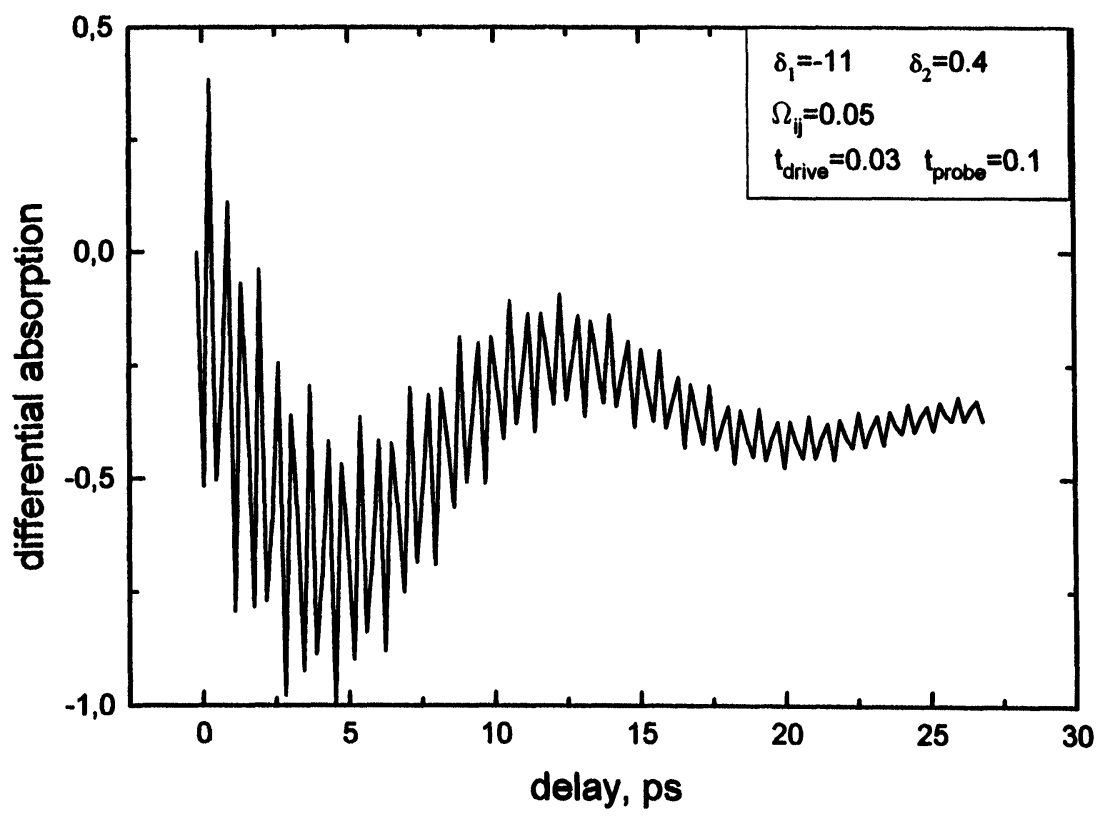

Figure 7 Differential absorption signal, initial conditions according to fig. 6, other parameters see insert.

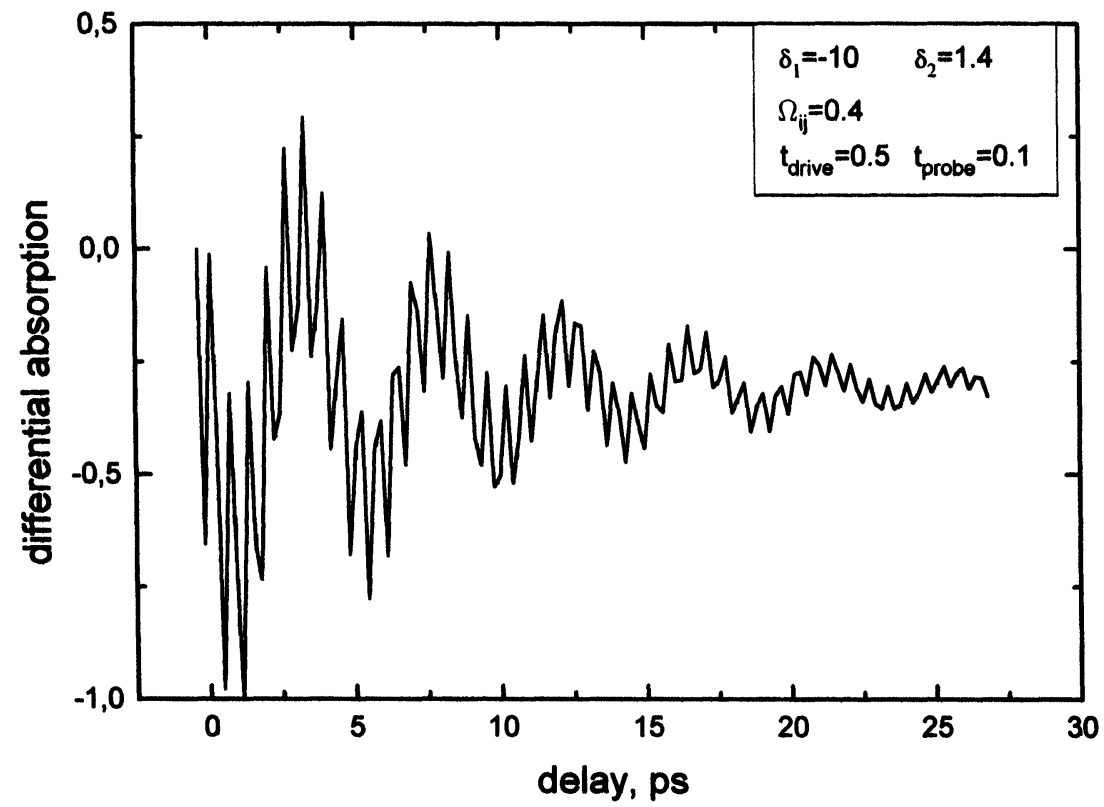

Figure 8 Differential absorption signal, initial conditions according to fig. 6, other parameters see insert. 


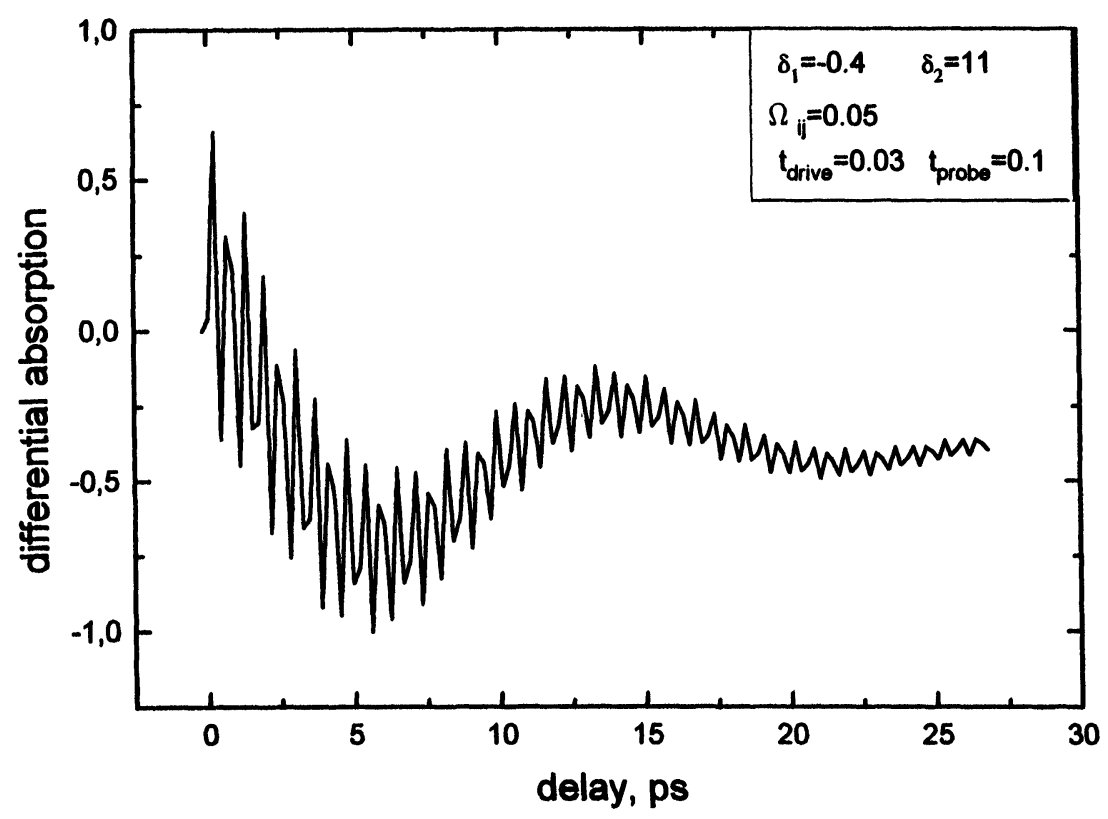

Figure 9 Differential absorption signal, initial conditions according to fig. 6, other parameters see insert.

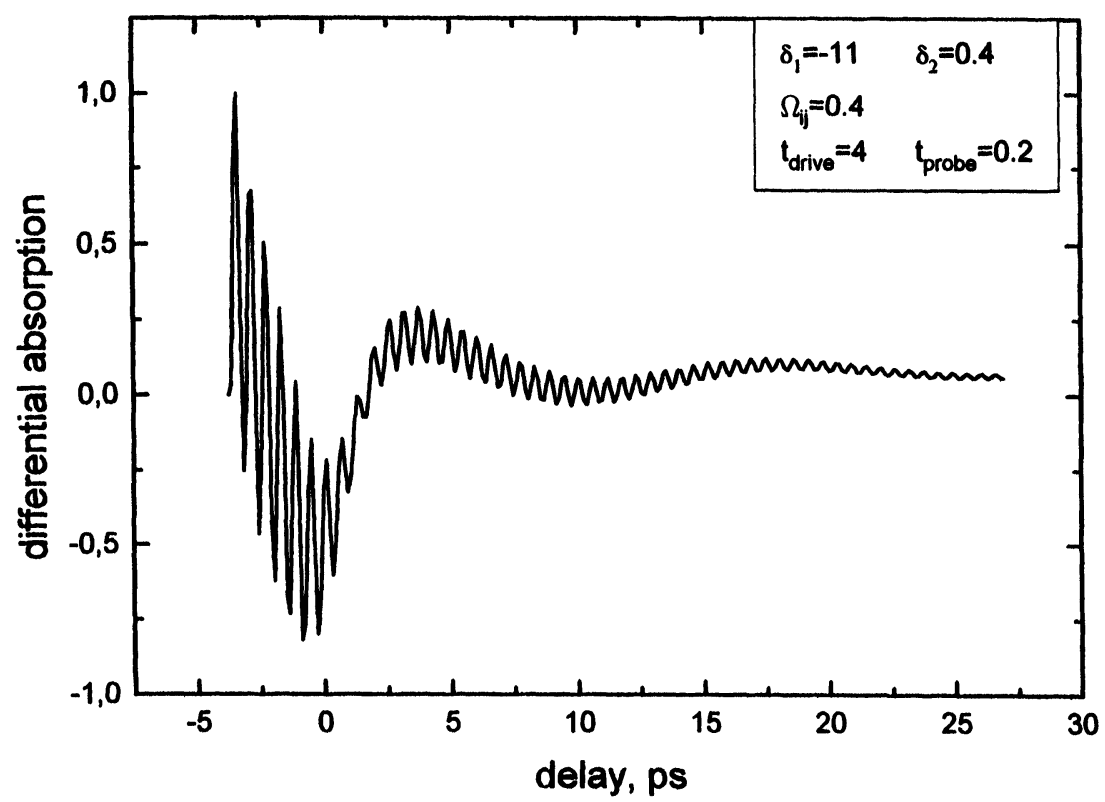

Figure 10 Differential absorption signal, initial conditions according to fig. 6, other parameters see insert. 


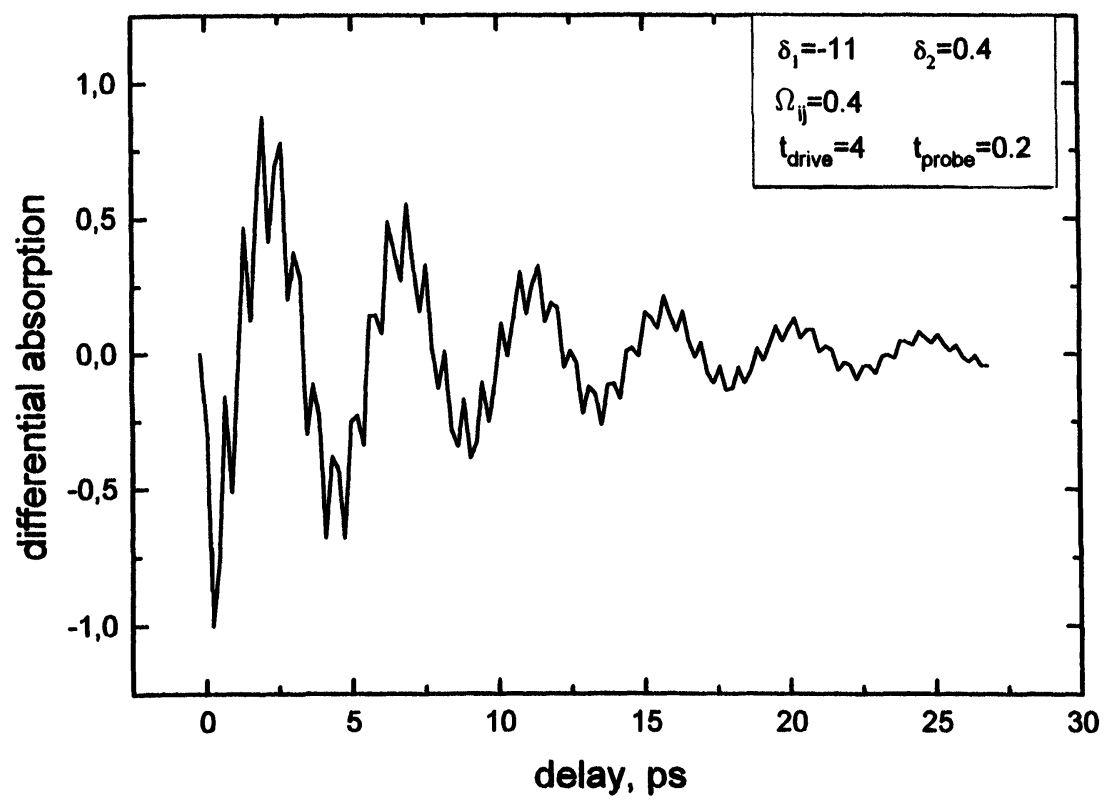

Figure 11 Differential absorption signal, initial conditions according to (2.24), other parameters see insert.

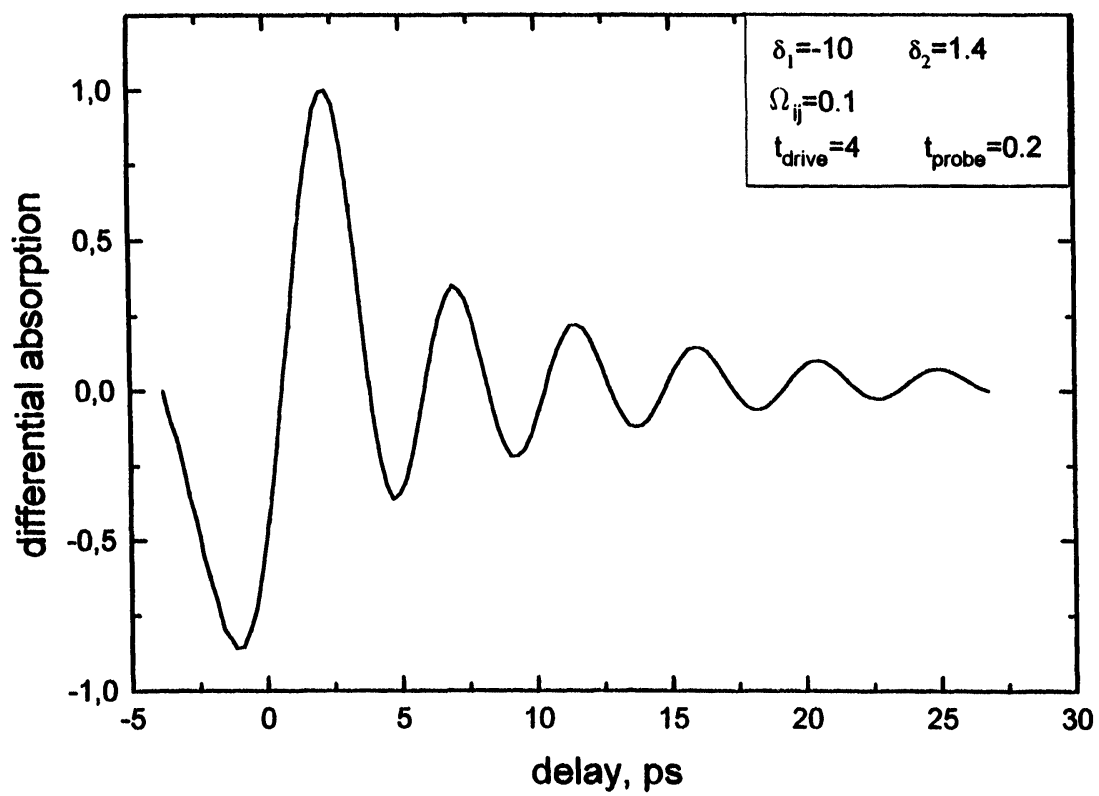

Figure 12 Differential absorption signal, initial conditions according to (2.24), other parameters see insert. 


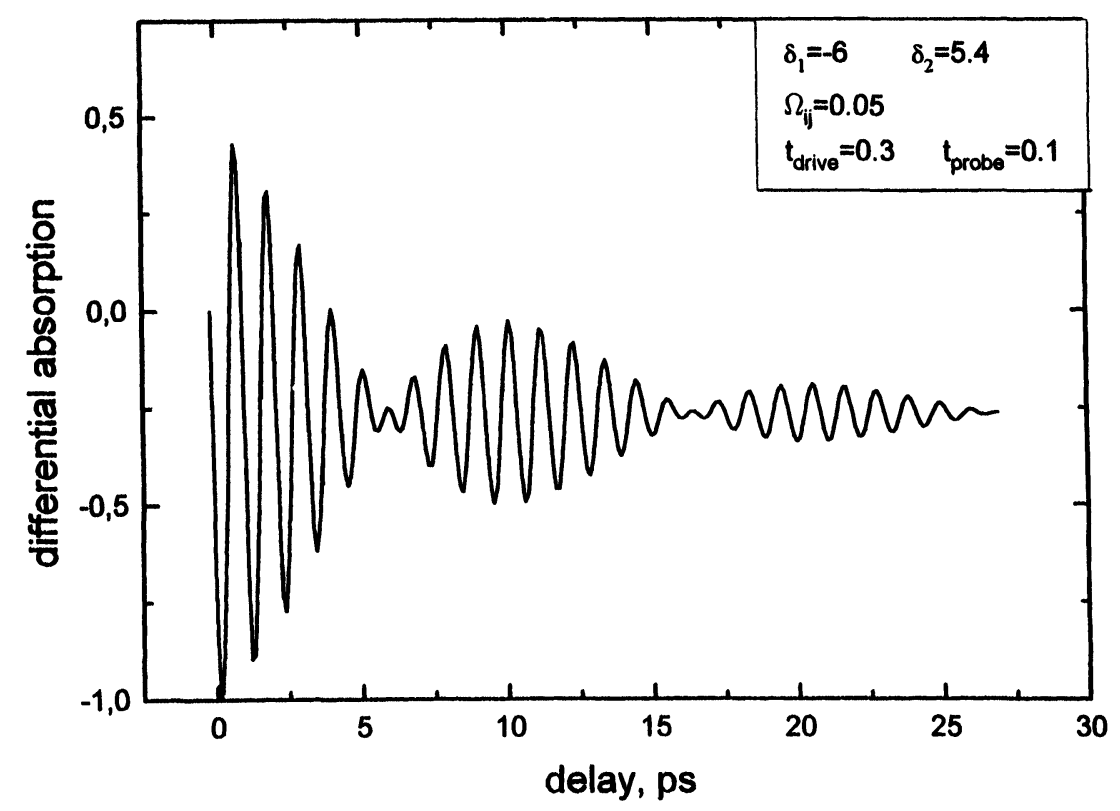

Figure 13 Differential absorption signal, initial conditions according to fig. 6, other parameters see insert.

emission with nearly equal frequencies. The observability of quantum beats is sensitive to the initial state the molecular system is prepared in previous to the driving and to the duration of the driving pulse. The quantum beats are generated most efficiently, if the system is initially prepared in a state, in which the occupation numbers of all three levels are comparable, and so are the absolute values of the non-diagonal density matrix elements; and if the driving pulses are not longer than some hundreds of femtoseconds. Otherwise stimulated emission induced by the driving pulse leads to a fast decrease of the occupation number of the upper levels, and at the same time of the polarisation corresponding to the less resonant transition, so that it holds

$$
\left|\tilde{\rho}_{12}(\mathrm{~T})\right| \ll\left|\tilde{\rho}_{23}(\mathrm{~T})\right|
$$

or

$$
\left|\tilde{\rho}_{12}(\mathrm{~T})\right| \gg\left|\tilde{\rho}_{23}(\mathrm{~T})\right|,
$$

and in (3.4) one oscillation decreases compared with the other one.

This behaviour is clarified by the occupation number behaviour, e.g. of $\rho_{33}(\mathrm{t})$. Its decrease consists of an intensity dependent part which is due to stimulated emission and a part with the time constant $\mathrm{k}_{31}$ due to relaxation.

b) Furthermore, the delay time range, in which quantum beats are observable, of course depends on the phase relaxation time in the system, as can be seen by (3.1-3.3). This is due to the decreasing of both oscillation amplitudes with the dephasing as the time constant. The temperature dependence of dephasing times in J-aggregates exhibits, that $T_{2}$ is in the fs range except at very low temperatures, 
where it is up to about $10 \mathrm{ps}$. Therefore quantum beats can be resolved only in the femtosecond delay regime, or, if it is wanted to observe them as long as possible, the sample has to be cooled down to a few $\mathrm{K}$.

\section{CONCLUSION}

In this paper, using a three-level model, we have shown, that under appropriate experimental conditions coherent transients are expected to be observable in large aggregate systems. As an example, we have calculated quantum beats in differential probe absorption measurements in PIC-J-aggregates in the ps time range, i.e. on a time scale comparable to the exciton-exciton annihilation characteristic time. Therefore it could be expected, that the vibrationally excited states, over which the excess energy is distributed by relaxation under such conditions, especially for very low temperatures, could possibly be driven coherently. In our forthcoming investigations we will include the exciton-exciton annihilation and relaxation process by a binary reaction model to clarify the character of these processes. Moreover, in a forthcoming paper we will modify the basic model to include the description of degenerate four-wave mixing experiments.

\section{Acknowledgements}

The authors thank Prof. Dr. A. Piskarskas, Vilnius for stimulating discussions.

\section{References}

1. M. J. Rosker, F. W. Wise and C. L. Tang, Phys. Rev. Lett., 37, (3) 321 (1986).

2. M. Gruebele and A. H. Zewail, Phys. Today, 24 May 1990.

3. V. Blanchet, M. -A. Bouchène, O. Cabrol and B. Girard, Chem. Phys. Lett., 233, 491 (1995).

4. D. M. Jonas, S. E. Bradforth, S. A. Passino and G. R. Fleming, J. Phys. Chem., 99, 2594 (1995).

5. Y. R. Shen, "The Principles of Nonlinear Optics", New York, (1984).

6. M. Mitsunaga and C. L. Tang, Phys. Rev., A35, (4), 1720 (1987).

7. I. A. Walmsley, M. Mitsunaga and C. L. Tang, Phys. Rev., A38 (9), 4681 (1988).

8. J. A. Leegwater, J. Phys. Chem., 99, 11605 (1995).

9. W. T. Pollard, S. -Y. Lee and R. A. Mathies, J. Chem. Phys., 92 (7), 4012 (1990).

10. A. Yasuda and T. Kobayashi, Chem. Phys. Lett., 209, (5-6), 531 (1993).

11. Z. H. Kafafi, J. R. Lindle, R. G. S. Pong, F. J. Bartoli, L. J. Lingg and J. Miliken, Chem. Phys. Lett., 188,492 (1992).

12. S. R. Flom, R. G. S. Pong, F. J. Bartoli and Z. H. Kafafi, Phys. Rev., B46, 15598 (1992).

13. E. E. Jelley, Nature 138, 1009 (1936).

14. G. Scheibe, Angew. Chem. 49, 563 (1936).

15. F. C. Spano and S. Mukamel, J. Chem. Phys., 95 (10), 7526 (1991).

16. H. Fidder, J. Knoester and D. A. Wiersma, J. Chem. Phys., 98 (8), 6564 (1993).

17. L. Valkunas, G. Trinkunas, V. Liuolia and R. van Grondelle, Biophys. J., 69, 1117 (1995).

18. F. C. Spano, J. R. Kuklinski and S. Mukamel, Phys. Rev. Lett., 65 (2), 211 (1990).

19. V. Sundström, T. Gillbro, R. A. Gadonas and A. Piskarskas, J. Chem. Physics, 89 (5), 2754 (1988).

20. H. S. Nalwa, Adv. Mater., 5 (5), 341 (1993).

21. T. Kobayashi, Nonlinear Optics 1, 91 (1991).

22. D. Möbius, Adv. Mater., 7 (5), 437 (1995).

23. K. Minoshima, M. Taiji, K. Misawa and T. Kobayashi, Chem. Phys. Lett., 218, 67 (1994). 
24. E. Gaižauskas and L. Valkunas, Opt. Commun. 109, 75 (1994).

25. E. Gaižauskas and L. Valkunas, Lietuvos fiezikos žurnalas, 34 (4), 384 (1994).

26. R. Gadonas, K. -H. Feller and A. Pugžlys, Opt. Commun. 112, 162 (1994).

27. R. Gadonas, K. -H. Feller and A. Pugžlys, J. Chem. Phys., submitted.

28. H. Stiehl, S. Dähne and K. Teuchner, J. Luminescence 39, 351 (1988).

29. S. De Boer and D. A. Wiersma, Chem. Phys. Lett., 165 (1), 45 (1990).

30. H. Fidder, J. Knoester and D. A. Wiersma, Chem. Phys. Lett., 171 (5-6), 529 (1990).

31. R. Gagel, R. Gadonas and A. Lauberau, Chem. Phys. Lett., 217 (3), 228 (1994). 